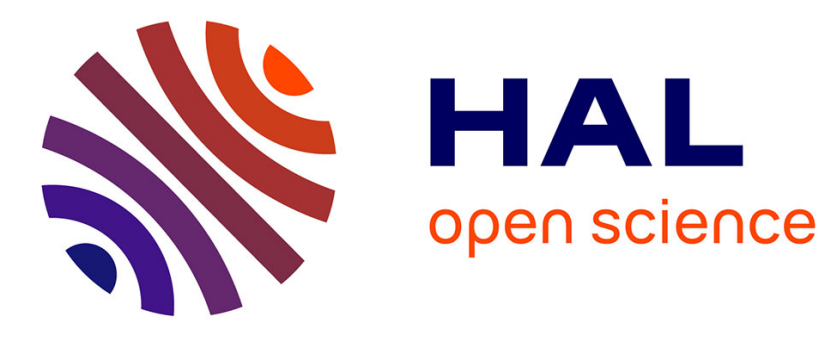

\title{
Passive guided wave tomography for structural health monitoring
}

Tom Druet, Arnaud Recoquillay, Bastien Chapuis, Emmanuel Moulin

\section{To cite this version:}

Tom Druet, Arnaud Recoquillay, Bastien Chapuis, Emmanuel Moulin. Passive guided wave tomography for structural health monitoring. Journal of the Acoustical Society of America, 2019, 146 (4), pp.2395-2403. 10.1121/1.5128332 . cea-02975905

\section{HAL Id: cea-02975905 https://hal-cea.archives-ouvertes.fr/cea-02975905}

Submitted on 23 Oct 2020

HAL is a multi-disciplinary open access archive for the deposit and dissemination of scientific research documents, whether they are published or not. The documents may come from teaching and research institutions in France or abroad, or from public or private research centers.
L'archive ouverte pluridisciplinaire HAL, est destinée au dépôt et à la diffusion de documents scientifiques de niveau recherche, publiés ou non, émanant des établissements d'enseignement et de recherche français ou étrangers, des laboratoires publics ou privés. 


\title{
Passive guided waves tomography for structural health monitoring
}

\author{
Tom Druet, ${ }^{1}$ Arnaud Recoquillay, ${ }^{1}$ Bastien Chapuis, ${ }^{1}$ and Emmanuel Moulin ${ }^{2}$ \\ ${ }^{1)}$ CEA, LIST, F-91191 Gif-sur-Yvette, France \\ ${ }^{2}$ IEMN UMR CNRS 8520, Université Polytechnique Hauts-de-France, F-59313 Valenciennes cedex 9 , \\ France
}

In this paper is presented a baseline-free quantitative method for imaging corrosion flaws in thin plates. It only requires an embedded guided wave sensor network used in a fully passive way, i.e. without active emission of waves. This method is called passive guided waves tomography. The aim of this development is the use of this method for the Structural Health Monitoring (SHM) of critical structures with heavy limitations on both sensor's intrusiveness and diagnostic's reliability because it allows to use sensors that cannot emit elastic waves such as Fiber BRAGG Gratings (FBGs) which are less intrusive than piezoelectric transducers (PZTs). The idea consists in using passive methods in order to retrieve the impulse response from elastic diffuse fields - naturally present in structures - measured simultaneously between the sensors. In this paper, two passive methods are studied: the ambient noise cross-correlation and the passive inverse filter. Once all the impulse responses between the sensors are retrieved, they are used as input data to perform guided wave tomography.

(C)2020 Acoustical Society of America. [ [http://dx.doi.org/10.1121/1.5128332]

$[\mathrm{XYZ}]$

Pages: $1-11$

\section{INTRODUCTION}

In most industries such as aeronautic, space, railway, shipbuilding, petrochemical or nuclear industry a failure can create a disaster, and thus asserting the structural integrity is critical. Hence, maintenance operations have a great impact on both costs and security.

The Structural Health Monitoring (SHM) consists in acquiring and analyzing data from sensors embedded in a structure in order to evaluate its health (SAE International, 2013). It allows to perform either scheduled maintenance, and thus to anticipate potential failures, or, in the long term, SHM-triggered maintenance. To that end, a SHM system must be able to provide a technical diagnostic with several levels of information on the flaw - detection, localization, classification, estimates of size - and then a technical prognostic in order to estimate the remaining lifetime of the structure.

Various defects needing to be taken care of exists in industrial applications. These defects can appear during the fabrication or during the life of the structure. The SHM generally addresses the issue of the inspection during the operational life of the structure. The flaws can either appear instantaneously or slowly progress over time. This paper deals with the latter kind of flaws such as corrosion or erosion.

A physical way to interrogate large and thin structures such as aircraft fuselages, pipes or ship hulls can be offered by guided elastic waves emitted and received by a network of piezoelectric transducers (PZTs) (Yu and

a) tom.druet@cea.fr
Giurgiutiu, 2008). Generally, the SHM methods based on elastic guided waves use a baseline. This baseline is compared to the current state by performing, for instance, a subtraction between signals in order to highlight the contribution of the defect. If the algorithms do not take into account external phenomena, these methods with a baseline are usually not robust to changes of environmental conditions. This may cause false alarms, which is very penalizing for SHM systems (Croxford et al., 2007). Many methods aim at correcting the effects of the changes of environmental conditions (Croxford et al., 2010; Liu et al., 2015; Putkis and Croxford, 2013) but, for now, they suffer from performance problems or seem to be impossible to implement in real structures.

In order to avoid the use of a baseline, it has been decided to increase the number of sensors used compared to classical solutions (Michaels, 2008; Quaegebeur et al., 2014; Zhao et al., 2007). This allows to get more information about the structure inspected, and thus to avoid the need of a baseline. Guided wave tomography algorithms are used here to that end. Moreover, this kind of algorithms are able to not only localize but also quantify the severity of defects consisting in a loss of thickness such as corrosion or erosion. However, increasing the number of sensors has a cost, particularly in terms of intrusiveness. A response to that issue could be to use undersampled data (Druet et al., 2019; Shi and Huthwaite, 2018) but, for now, the number of sensors' reduction achieved is of a factor two to four with respect to the optimal number. This reduction seems to be not enough for some industrial sectors such as aeronautic industry which has a tremendous added mass constrain. 
Another interesting possibility is to design a guided wave based SHM system with minimal intrusiveness thanks to the use of fiber BRAGG gratings (FBGs). However, these sensors cannot emit waves, they can only work in a passive way. This means that passive methods are needed to retrieve the response between two sensors. Indeed, in the past years researchers have shown that it is possible to use ambient structure-borne elastic noise instead of actively emit the waves in the structure in order to retrieve the impulse response of the medium between two sensors (Sabra et al., 2007a). Moreover, it has been shown in (Druet et al., 2018) that passive guided wave measurements are possible thanks to the coupling between FBGs and passive methods. In this paper is shown how to perform passive tomography by coupling guided wave tomography adapted to SHM framework (Druet et al., 2019) with passive methods. Finally, the passive methods have several other advantages in the context of SHM (Chehami et al., 2014; Sabra et al., 2008). First, since it is not necessary to inject energy in the structure for wave emission, the power consumption of the SHM system can be significantly reduced. This could be of crucial importance for embedded systems with limited or no access to power sources like aircraft or underwater monitoring systems. Then, the complexity of both the electrical cabling and the embedded electronics are reduced, since no emission circuitry is necessary.

This paper shows in a first section the methodology to perform passive guided wave tomography. Two passive methods are presented: the first one is called "ambient noise cross-correlation" and the second one is called "passive inverse filter". Then some keys presented in (Druet et al., 2019) to adapt guided waves tomography to the SHM framework are recalled. Finally, comparisons between active and passive signals are presented and passive guided wave tomographies for both passive methods are studied for an ambient noise respecting more or less the equipartition hypothesis.

\section{METHODS}

In this section is presented how to perform a passive guided wave tomography. First the basis of the two passive methods used in this paper are given and then the main steps of guided wave tomography are recalled.

\section{A. Passive methods: from cross-correlation to passive inverse filter}

The first step of any imaging algorithm is the data acquisition. This step usually consists in successively emitting a wave with a sensor and measuring the signal with all others. These signals are called in the following active signals. Another way to obtain equivalent data is to measure the ambient noise at all sensors simultaneously and then process it to reconstruct the data used in the imaging algorithm. This method is called in the following passive acquisition and the corresponding signals are called passive signals.
A few algorithms exist to retrieve the data from the ambient noise. The most known is the ambient noise cross-correlation (Lobkis and Weaver, 2001), which has been applied in various domains (Campillo and Paul, 2003; Davy et al., 2016; Duvall Jr et al., 1993; Lobkis and Weaver, 2001; Roux et al., 2004; Sabra et al., 2007a’b). This method is fully justified in many cases, but some hypotheses are needed concerning the noise spatial repartition, and it is well known that the quality of the reconstruction is strongly linked to the fulfilment of these hypotheses. To increase the quality of the reconstruction, it is then possible to use the correlation of the coda of the noise cross-correlation (Stehly et al., 2008): when many sensors are involved in the acquisition, this method enhance the spatial distribution of the signal by using the coda of all reconstructed signals.

Another possibility is the passive inverse filter (Gallot et al., 2012), which is well suited when many receivers are used as in the case of the guided-wave tomography. As the passive inverse filter is based on the inversion, after regularization, of the noise response matrix, it is a global process, which reconstructs all passive signals from all measured ambient noises simultaneously. In this section will be described the passive inverse filter. To the authors' knowledge, a proof of this method is still an open subject which would give interesting insights on its necessary hypotheses. The description below gives the idea of the method.

In all the following, we consider linear acoustic waves. For a given source $f$, the acoustic field $\varphi$ in the domain $\Omega \subset \mathbb{R}^{d}, d \in\{2,3\}$, is solution to

$$
\frac{\partial^{2} \varphi}{\partial t^{2}}-\frac{1}{c^{2}} \Delta \varphi=f, \quad \text { in } \Omega \times \mathbb{R},
$$

to which is added relevant initial and boundary conditions.

First of all, let us start with a short recall of the ambient noise cross-correlation. Consider a set of point sensors placed at $\left(\boldsymbol{x}_{j}\right), j=0, \ldots, N-1$. The empirical cross-correlation of the signals $u$ measured over the time interval $[0, T], T>0$, at $\boldsymbol{x}_{i}$ and $\boldsymbol{x}_{j}$ is

$$
C\left(\boldsymbol{x}_{i}, \boldsymbol{x}_{j}, t ; T\right)=\frac{1}{T} \int_{0}^{T} u\left(\boldsymbol{x}_{i}, \tau\right) u\left(\boldsymbol{x}_{j}, \tau+t\right) \mathrm{d} \tau .
$$

Suppose now that the source term $f$ is an unknown noise source, which is a space-time stationary random field that is also delta correlated in space, which means that, for all $t, \tau>0$ and points $\boldsymbol{x}$ and $\boldsymbol{y}$, the following relation holds

$$
\langle f(\boldsymbol{x}, t) f(\boldsymbol{y}, \tau)\rangle=K(\boldsymbol{x}) \delta(\boldsymbol{x}-\boldsymbol{y}) F(t-\tau),
$$

where $\langle\cdot\rangle$ denotes the ensemble average, $K$ is a function characterizing the spatial distribution of the noise sources and $F$ is the normalized time correlation function, its Fourier transform being linked to the spectral energy density of the ambient noise. A common further assumption to be able to recover active signals from passive acquisitions using the cross-correlation is then that 
the ambient noise is well distributed in space, that is $K \equiv 1$ over a suitable subdomain of $\Omega$, typically all $\Omega$. This last assumption combined with (3) is often refered to as the equipartition hypothesis. It can then be proved (Garnier and Papanicolaou, 2016) that

$\lim _{T \rightarrow+\infty} \frac{\mathrm{d} C}{\mathrm{~d} t}\left(\boldsymbol{x}_{i}, \boldsymbol{x}_{j}, t ; T\right) \propto F *\left[G\left(\boldsymbol{x}_{i}, \boldsymbol{x}_{j}, \cdot\right)-G\left(\boldsymbol{x}_{i}, \boldsymbol{x}_{j},-\cdot\right)\right](t)$

where $*$ denotes the convolution operation in time and $G\left(\cdot, \boldsymbol{x}_{j}, \cdot\right)$ is the Green function of the problem, that is the solution of (1) with $f=\delta\left(\cdot-\boldsymbol{x}_{j}\right)$. An interesting fact about the cross-correlation is its link to the time reversal operator (Gallot, 2010): consider the Fourier transform of the limit of the empirical cross-correlation (2) as $T$ goes to infinity,

$$
\widehat{C}\left(\boldsymbol{x}_{i}, \boldsymbol{x}_{j}, \omega\right)=\widehat{u}\left(\boldsymbol{x}_{i}, \omega\right) \overline{\widehat{u}\left(\boldsymbol{x}_{j}, \omega\right)} .
$$

For a given frequency $\omega$, define the cross-correlation ma$\operatorname{trix} \mathcal{C}$ as

$$
\mathcal{C}_{i j}=\widehat{C}\left(x_{i}, x_{j}, \omega\right)
$$

This matrix is then equal to the time-reversal operator, which would equal to $\boldsymbol{u} \boldsymbol{u}^{*}$, where $\boldsymbol{u}$ is the signal vector, the coefficients of which are the Fourier transform of the measurements at each sensor. Consider now that $P$ noise acquisitions are done or equivalently if $T$ is big enough, that the acquired ambient noise can be cut into $P$ parts, $P>N$. Instead of $\boldsymbol{u}$, consider $\mathcal{U}$ which is a $N \times P$ matrix containing all noise acquisitions. The link between the cross-correlation matrix and the time-reversal operator holds:

$$
\mathcal{C}=\mathcal{U U}^{*}
$$

This enables the introduction of the passive inverse filter: instead of considering $\mathcal{U} \mathcal{U}^{*}$, the passive inverse filter consists in right-multiplying $\mathcal{U}$ by a regularized inverse of $\mathcal{U}$, namely $\tilde{\mathcal{U}}^{-1}$. More precisely, the regularization is done thanks to a singular value decomposition, in which only the biggest singular values are kept, which will be explained at the end of this section. If we denote $\mathcal{U}=\mathcal{V D} \mathcal{W}$ the singular value decomposition of $\mathcal{U}$, where $\mathcal{V}$ and $\mathcal{W}$ are unitary matrices containing the left and right singular vectors and $\mathcal{D}$ is a diagonal matrix containing the singular values of $\mathcal{U} \sigma_{i}$ sorted in descending order, then

$$
\mathcal{U} \tilde{\mathcal{U}}^{-1}=\mathcal{V}\left(\begin{array}{cc}
I_{M} & 0 \\
0 & 0
\end{array}\right) \mathcal{V}^{*}
$$

where $M<N$ is the number of singular values kept after regularization. Again, this operator is close to the timereversal one:

$$
\mathcal{U U}^{*}=\mathcal{V} \mathcal{D}_{N} \mathcal{V}^{*}
$$

where $\mathcal{D}_{N}$ is a $N \times N$ diagonal matrix of coefficients $\left|\sigma_{i}\right|^{2}$. Hence, the passive inverse filter can be seen as a regularized version of the cross-correlation (Seydoux et al., 2017).

The difference between the two operators is the relative weight given to each singular direction. Whereas the time-reversal operator gives a bigger weight to the first singular directions, with a quick decrease due to the squared singular values, the passive inverse filter gives the same weight to all selected singular directions, which seems to enable the extraction of pieces of information from the ambient noise which would be neglected otherwise. In the same way, the normalization at each frequency implies that they all have the same weight in the final reconstructed signal, which induces a whitening of the reconstructed signal. The difficulty is then to correctly choose the singular directions of interest, which will be kept in the regularized inverse of $\mathcal{U}$. To do so, a classic technique is to consider the variations of the modulus of the singular values: a usual behavior consists in a fast decay followed by a slower decay. The point of the change of slope is a good truncation candidate (Gallot et al., 2012) and will be used in our experimental study.

\section{B. Guided wave tomography}

In this article, the used guided wave tomography algorithm is based on the original iterative Hybrid Algorithm for Robust Breast Ultrasound Tomography (HARBUT) (Huthwaite and Simonetti, 2013) but adapted to the heavy constraints imposed by the SHM framework. This adaptation has been detailed in a previous work (Druet et al., 2019). The same algorithm is used here to produce images of corrosion-like defects but this time using passive data. In this section are recalled the main elements of the imaging algorithm and a regularization method called "variable relaxation" which help to improve significantly the quality of the image when undersampled data are used.

In all the following, a single mode is considered. The wavenumbers and velocities used in this section are implicitly those of this mode. The iterative version of HARBUT includes the following principal steps. In order to initialize the algorithm, a time-of-flight tomography image of the plate thickness is computed in order to fulfill the Born approximation in the subsequent diffraction tomography step. For the time-of-flight tomography, the structure to be imaged is represented at the position $\boldsymbol{x}$ by the scalar slowness field $s(\boldsymbol{x})=1 / v_{\mathrm{G}}(\boldsymbol{x})$, where $v_{\mathrm{G}}$ is the group velocity of the considered mode. To represent the slowness $s(\boldsymbol{x})$, we project it on a finite basis. The projection of the scalar slowness $\tilde{s}(\boldsymbol{x})$ thus reads

$$
\tilde{s}(\boldsymbol{x})=\sum_{i, j} S_{i j} \beta_{i j}(\boldsymbol{x}),
$$

where $\beta$ is a $2 \mathrm{D}$ B-spline basis and $S_{i j}$ is the coefficient associated to the $\mathrm{B}$-spline $\beta_{i j}$. The slowness is then linked to the time-of-flight $\tau_{k \rightarrow l}$ for a trajectory $\gamma_{k \rightarrow l}$ between the emitter $k$ and the receiver $l$ :

$$
\tau_{k \rightarrow l}=\sum_{i, j} S_{i j} A_{i j k l},
$$


where $A_{i j k l}=\int_{\gamma_{k \rightarrow l}} \beta_{i j}(\gamma) \mathrm{d} \gamma$ is the contribution of the basis element $\beta_{i j}$ to the time-of-flight $\tau_{k \rightarrow l}$. The image is then updated thanks to an absolute estimator based on the difference between the data $\tau_{k \rightarrow l}^{\text {defect }}$ and the computed time-of-flight $\tau_{k \rightarrow l}^{(n-1)}$ on the $(n-1)^{\text {th }}$ map. The $\mathrm{n}^{\text {th }}$ iteration of the time-of-flight tomography step reads: $S_{i j}^{(n)}=S_{i j}^{(n-1)}+\delta_{k} S_{i j}^{(n)}$, where $\delta_{k} S_{i j}^{(n)}$ is the correction applied to the coefficient $S_{i j}$ and is computed according to the following formulation:

$$
\delta_{k} S_{i j}^{(n)}=\frac{1}{N_{i j k}} \sum_{l}\left(W_{i j k l} \frac{\tau_{k \rightarrow l}^{\text {defect }}-\tau_{k \rightarrow l}^{(n-1)}}{\lambda_{k \rightarrow l}}\right)
$$

where $\lambda_{k \rightarrow l}$ is the trajectory's length, $W_{i j k l}$ is a weighting of the path, consisting of $A_{i j k l}$ in the simplest case but usually including a Hamming window to avoid side effects, and the normalization factor $N_{i j k}$ reads: $N_{i j k}=$ $\sum_{l} A_{i j k l}$.

Once the time-of-flight tomography image is computed, it becomes the first guess of an iterative diffraction tomography process. The image is then represented thanks to an object function $\mathrm{O}(\boldsymbol{x})$, which reads

$$
\mathrm{O}(\boldsymbol{x})=k(\boldsymbol{x})^{2}-k_{0}^{2}=k_{0}^{2}\left(\left(\frac{v_{0}}{v(\boldsymbol{x})}\right)^{2}-1\right)
$$

where $k_{0}$ is the background wavenumber, $v_{0}$ the background phase velocity and $v(\boldsymbol{x})$ the phase velocity at the position $\boldsymbol{x}$, which is linked to the thickness thanks to the dispersion curves when the algorithm has converged. One can show (Huthwaite, 2016a) that $\mathrm{O}(\boldsymbol{x})$ reads

$$
\mathrm{O}(\boldsymbol{x})=\int_{-\pi}^{\pi} \int_{-\pi}^{\pi} \frac{\widehat{\varphi}_{\boldsymbol{k}_{0}}^{s}\left(\boldsymbol{x}_{l}\right)}{G_{0}\left(\boldsymbol{x}_{l} ; \boldsymbol{x}\right) G_{0}\left(\boldsymbol{x} ; \boldsymbol{x}_{k}\right)} \mathrm{W}\left(\theta_{k}, \theta_{l}\right) \mathrm{d} \theta_{k} \mathrm{~d} \theta_{l}
$$

where $\widehat{\varphi}^{s}$ is the scattered field, $G_{0}$ the GREen's function of the unperturbed domain, $\mathrm{W}$ a weighting function coming from a variable change, $\boldsymbol{x}_{k}$, respectively $\boldsymbol{x}_{l}$, the position of the emitter $k$, respectively the receiver $l$, and $\theta_{i}, i \in\{k, l\}$, the angles of the emitter and receiver. Note that it is possible (Huthwaite, 2016a) to replace scattered field $\widehat{\varphi}^{s}$ by the total field $\widehat{\varphi}$. Iterative HARBUT consists in computing iteratively the correction $\mathrm{O}_{\delta}(\boldsymbol{x})$, which is added to the previous estimation $\mathrm{O}_{b}(\boldsymbol{x})$ so that $\mathrm{O}(\boldsymbol{x})=\mathrm{O}_{b}(\boldsymbol{x})+\mathrm{O}_{\delta}(\boldsymbol{x})$.

More details about original HARBUT can be found in (Huthwaite and Simonetti, 2013) but also in (Huthwaite, 2014' 2016a'b'c; Huthwaite et al., 2013) for many following developments. Concerning the adaptations to the constraints imposed by the SHM framework which are used also in this paper, they can be found in (Druet et al., 2019). We recall here one of these adaptations which concern the regularization called "variable relaxation" and which allows to limit the amplification of spatial fluctuations at each iteration due to the noise and the lack of sensors, while still allowing us to accurately reconstruct localized defects above the noise threshold. Let $e_{i j}$ and $\tilde{e}$ be two parameters depending on the used algorithm. More precisely, let $e_{i j}$ be the difference between the computed thickness and the pristine one denoted $\tilde{e}$ for the time-of-flight tomography, and let $e_{i j}$ be the object function $\mathrm{O}$ and $\tilde{e}=-\min \mathrm{O}$ for the diffraction tomography. Let $e_{i j}^{\prime}$ be the new considered value at the position $i j$. The "variable relaxation" is defined as follows:

$$
e_{i j}^{\prime}= \begin{cases}\frac{e_{i j}}{\left(1+\frac{1}{z_{i j}^{2}}\right)^{\frac{\alpha}{2}}} & \text { if }\left|e_{i j}\right|<\gamma \beta \tilde{e} \\ e_{i j} & \text { otherwise }\end{cases}
$$

with $\beta$ a threshold depending on the amplitude fluctuation of the noise level of the image, $\gamma$ a constant determining the relaxation limit, $\alpha$ a constant defining the strength of the regularization and $z_{i j}$ reads

$$
z_{i j}= \begin{cases}\frac{\left|e_{i j}\right|}{\beta \tilde{e}} & \text { if }\left|e_{i j}\right|<\beta \tilde{e}, \\ \frac{\left|e_{i j}\right|}{\frac{1}{2} \beta \tilde{e}\left[1-\cos \left(\pi \frac{\left|e_{i j}\right|-\gamma \beta \tilde{e}}{(1-\gamma) \beta \tilde{e}}\right)\right]} & \text { if } \beta \tilde{e} \leq\left|e_{i j}\right|<\gamma \beta \tilde{e} .\end{cases}
$$

As explained in (Druet et al., 2019), we chose to take $\gamma \beta=1, \beta=0.1, \alpha=4$ for the time-of-flight tomography and $\alpha=2$ for the diffraction tomography.

\section{RESULTS}

In this section, we present experimental results on passive tomography. First, the experimental setup is presented. Then the differences between the crosscorrelation and the passive inverse filter presented in the section II A are illustrated with classical signal processing tools. In order to confirm the good convergence of passive methods, passive and active signals are compared for several distances in the following section. Finally, we present passive guided wave tomography images for both cross-correlation signals and passive inverse filter signals.

\section{A. Experimental setup}

The experimental setup used to obtain all the results of this paper is shown in figure 1 . We consider an aluminum plate of density $\rho=2.7 \mathrm{~g} . \mathrm{cm}^{-3}$, longitudinal velocity $V_{\mathrm{L}}=6360 \mathrm{~m} . \mathrm{s}^{-1}$, transversal velocity $V_{\mathrm{T}}=$ $3140 \mathrm{~m} . \mathrm{s}^{-1}$ and dimensions $1400 \mathrm{~mm} \times 1250 \mathrm{~mm} \times 2 \mathrm{~mm}$. On this aluminum plate are glued 30 piezoelectric transducers of diameter $\varnothing=18 \mathrm{~mm}$ using epoxy glue. These sensors are equally distributed on a circle of radius $r=$ $300 \mathrm{~mm}$.

The ambient noise required to perform passive tomography has been generated in the plate by manually spraying compressed air randomly on its surface (Druet et al., 2018; Larose et al., 2007). The distance between the nozzle and the plate was of approximately $5 \mathrm{~cm}$. Ten seconds sampled at $2.5 \mathrm{MHz}$ of this ambient noise were recorded and then post-processed in order to obtain passive signals for all the couples of sensors. 


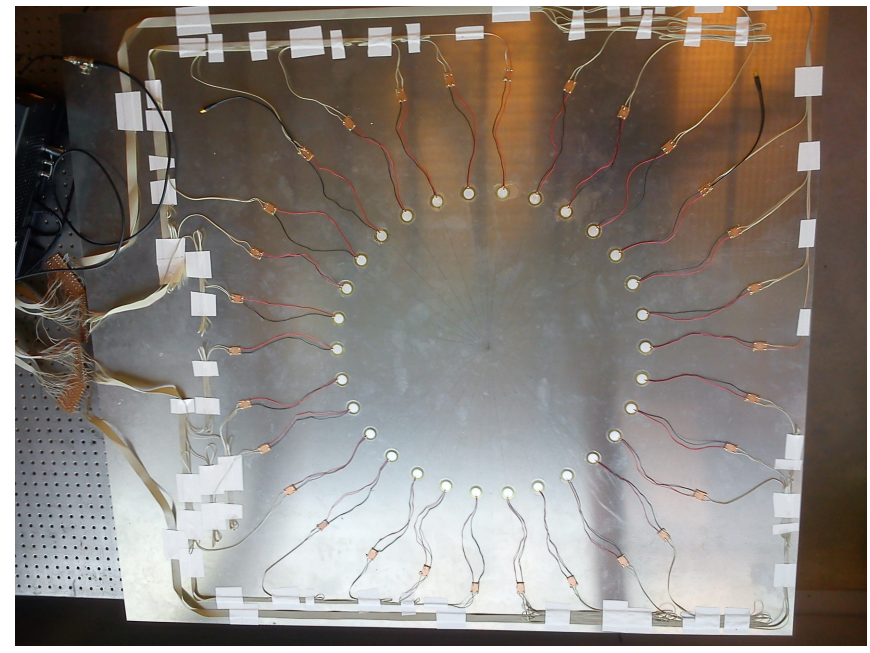

FIG. 1. (Color online) Aluminum plate and piezoelectric transducers positions.

\section{B. Cross-correlation versus Passive inverse filter}

In this section are illustrated the differences between the cross-correlation and the passive inverse filter on typical passive signals.

First, we present raw passive signals in figure 2 for a distance between sensors of $52 \mathrm{~cm}$. The case of the crosscorrelation is presented in figure 2(a) whereas the case of the passive inverse filter is presented in 2(b). We also present in figure 3 there spectral content.

Concerning the cross-correlation, we can identify wave packets around $400 \mu \mathrm{s}$ for the causal part and $-400 \mu \mathrm{s}$ for the anticausal one. They correspond approximately to the theoretical time-of-flight of the $\mathrm{A}_{0}$ mode for low frequencies, around $20 \mathrm{kHz}-30 \mathrm{kHz}$. This seems to be consistent because the spectral content of the crosscorrelation is centered around $25 \mathrm{kHz}$ as can be seen figure 3. Moreover, the cross-correlation of figure 2(a) is globally symmetrical, which is expected from the passive reconstruction and is an indication of a good reconstruction.

Concerning, the passive inverse filter, we would like to recall that the 30 sensors where used altogether to obtain the passive signal between the two sensors of figure 2(b) as explained in section II A. It is not possible to identify specific wave packets on the passive signal of figure 2(b) because of the whitening effect of the passive inverse filter: indeed, as can be seen figure 3, the spectral density of the passive inverse filter has a wider frequency range and the spectral density is somewhat constant over this frequency range, whereas the signal reconstructed thanks to the cross-correlation has the same energy repartition as the elastic noise. Thus high-frequency components, including electromagnetic noise, will contribute to the passive signal resulting from the inverse filter process. This noise induces significant autocorrelation peak at zero time as can be observed in figure 2(b). Since this peak, as well as the whole part of the signal be-

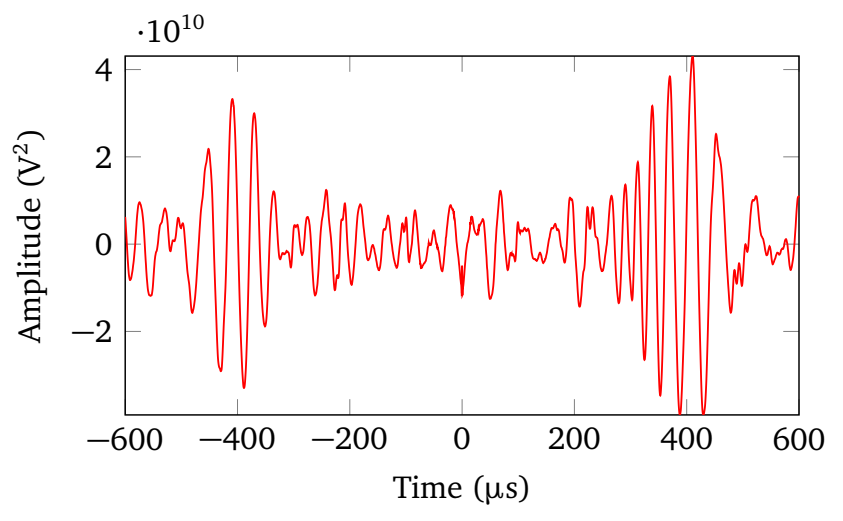

(a)

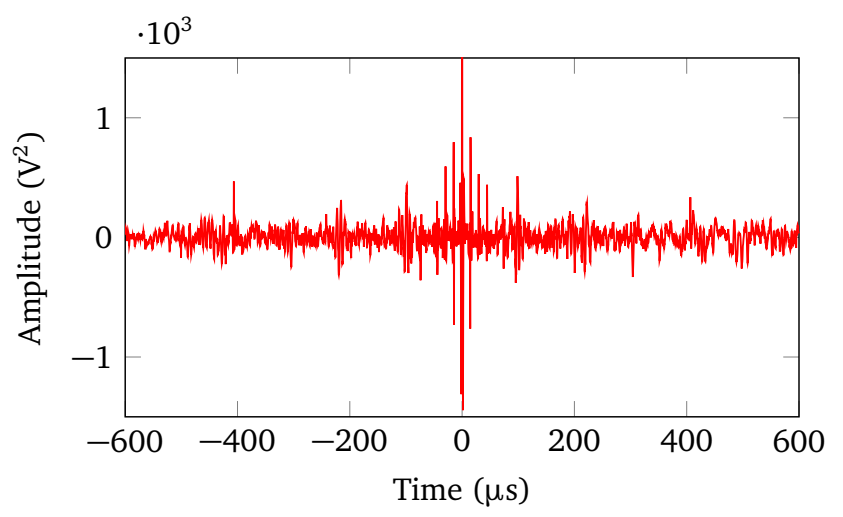

(b)

FIG. 2. Raw signals of (a) cross-correlation and (b) passive inverse filter.

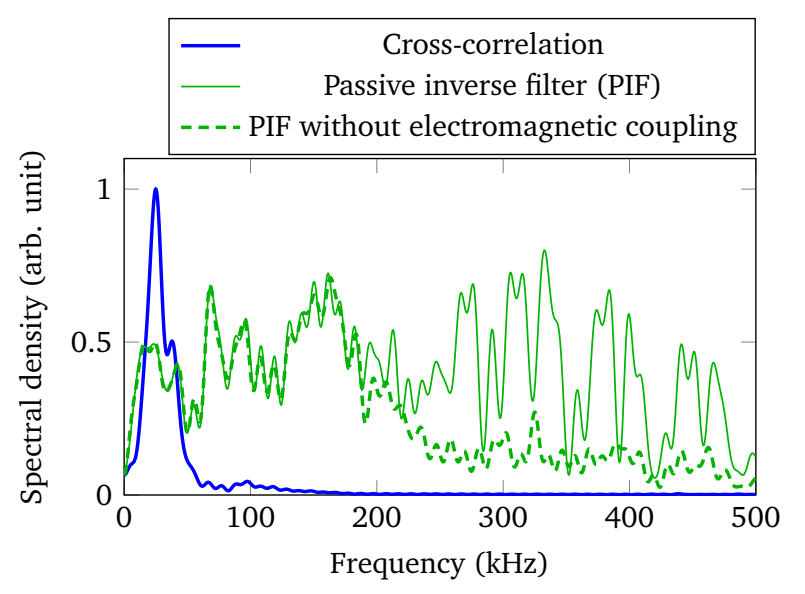

FIG. 3. (Color online) Spectral whitening effect of the passive inverse filter. 
fore the first modal wavepacket arrival (at approximately $100 \mu \mathrm{s}$ ), does not contain useful physical information, it is set to zero between $-65 \mu \mathrm{s}$ and $65 \mu \mathrm{s}$. This operation eliminates undesirable electromagnetic noise, while keeping the information concerning the guided modes. The figure 3 shows that the electromagnetic coupling has its energy at frequencies higher than $150 \mathrm{kHz} / 200 \mathrm{kHz}$.

In order to compare the physical information of the passive signals, the signals are projected in a time-frequency plan thanks to a reassigned spectrogram (Auger and Flandrin, 1995) presented in figure 4. The same experiment as before has been conducted but with a larger bandwidth in order to highlight the whitening effect of the passive inverse filter. To this end, we used two piezoelectric transducers of diameter $13 \mathrm{~mm}$ spaced $60 \mathrm{~cm}$ apart from each other. The reassigned spectrogram corresponding to the cross-correlation signal is plotted figure 4(a) and the one corresponding to the passive inverse filter is plotted figure 4(b). Once again it can be seen that the passive inverse filter has a whitening effect compared to the cross-correlation. Moreover, the power spectral density in both cases corresponds to the theoretical time-of-flight (red dash-line) of the $\mathrm{A}_{0}$ mode.

In both cases, the reconstructed signals are coherent with the theoretical ones. Signals reconstructed thanks to the passive inverse filter have a wider frequency range, which may be of interest for applications. It should be noted that the influence of the spatial repartition of the noise could not be fully studied in this case as the used plate was very reverberant, inducing a good spatial repartition of the ambient noise even for a localized noise source.

\section{Passive and active signals comparisons}

In this section are presented comparisons between active and passive signals for the frequency range of interest of this paper and for both the cross-correlation and the passive inverse filter.

Active signals $s(\boldsymbol{A}, \boldsymbol{B}, t)$ are obtained by emission of a 5 cycles toneburst at $20.4 \mathrm{kHz}$ from a piezoelectric transducer placed in $\boldsymbol{A}$. The receiver is placed in $\boldsymbol{B}$. The elastic guided waves are measured after their propagation for several distances between the emitter and the receiver (see figure 5). In order to get the same spectral density for passive signals named $s^{\mathrm{P}}(\boldsymbol{A}, \boldsymbol{B}, t)$ and active signals $s(\boldsymbol{A}, \boldsymbol{B}, t)$, the following normalization is performed (Druet et al., 2018):

$$
\widehat{s}^{\mathrm{P}}(\boldsymbol{A}, \boldsymbol{B}, \omega)=\left\{\begin{array}{lr}
\frac{\widehat{C}(\boldsymbol{A}, \boldsymbol{B}, \omega)}{|\widehat{C}(\boldsymbol{A}, \boldsymbol{B}, \omega)|}|\widehat{s}(\boldsymbol{A}, \boldsymbol{B}, \omega)| & \omega \in \Omega_{\varepsilon}, \\
\widehat{C}(\boldsymbol{A}, \boldsymbol{B}, \omega) & \text { otherwise, }
\end{array}\right.
$$

where $\hat{r}$ denotes the Fourier transform of a variable and the set $\Omega_{\varepsilon}$ reads $\Omega_{\varepsilon}=$ $\left\{\omega \in \mathbb{R}|| \widehat{C}(\boldsymbol{A}, \boldsymbol{B}, \omega)\left|>\varepsilon \max _{\omega}\right| \widehat{C}(\boldsymbol{A}, \boldsymbol{B}, \omega) \mid\right\}$, with $\varepsilon$ a threshold taken as $\varepsilon=0.01$ here. It should be noted that Equation (17) ensures the same power spectral

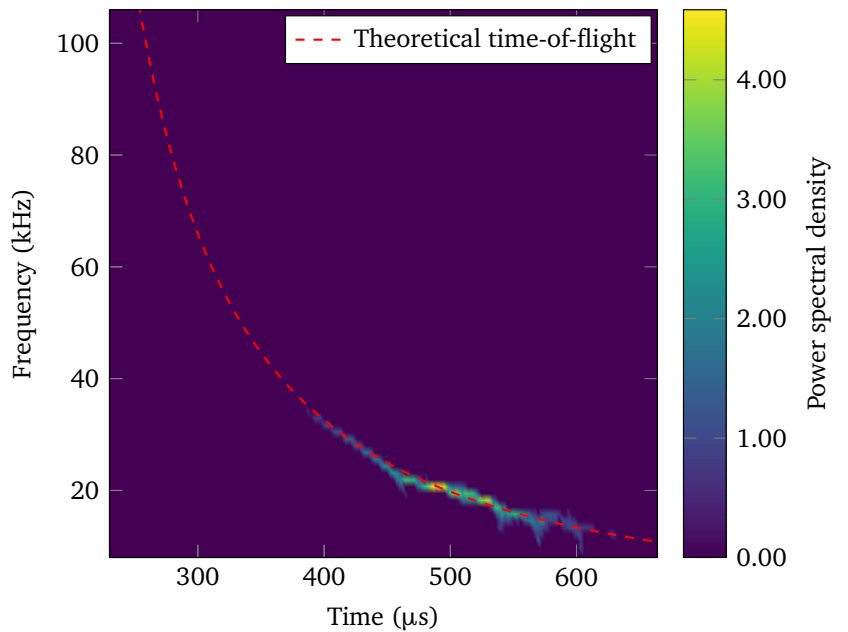

(a)

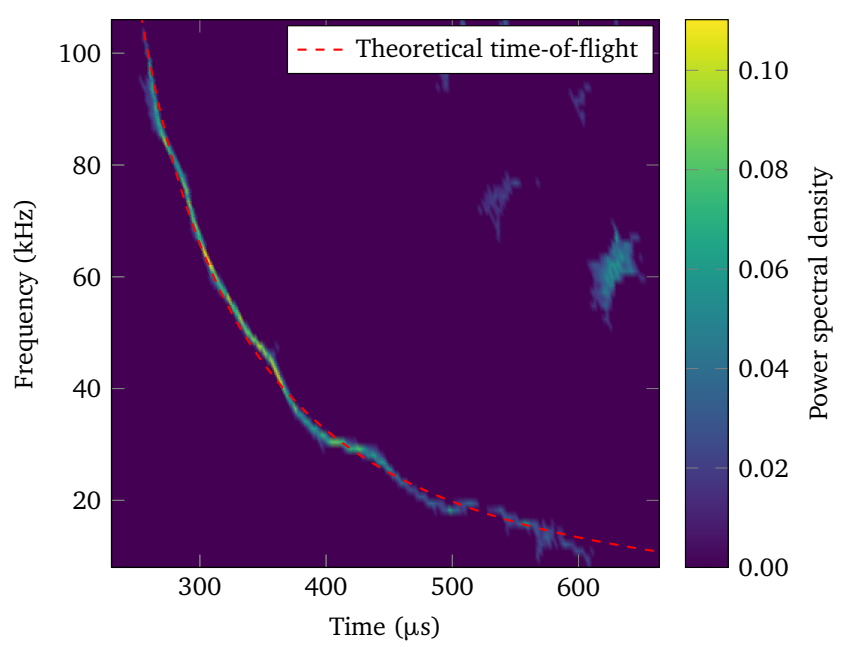

(b)

FIG. 4. (Color online) Reassigned spectrogram of (a) crosscorrelation raw signal and (b) Passive inverse filter raw signal.

density between active and passive signals but leaves the phase of the signals unchanged.

The comparison between the cross-correlation signals and the active signals is plotted figure 5(a) and the one concerning the passive inverse filter is plotted figure 5(b). For both cases, a very good match is observed. The theoretical time-of-flight is also plotted and corresponds to the maximum of the envelop of the wave packet corresponding to the $\mathrm{A}_{0}$ mode. Note that in both cases (figure $5(\mathrm{a})$ and $5(\mathrm{~b})$ ) the electromagnetic coupling around the time $t=0 \mu \mathrm{s}$ is observed.

The study in this section allows us to conclude that the passive signals seems to be of adequate quality to perform passive imaging and more precisely passive guided 


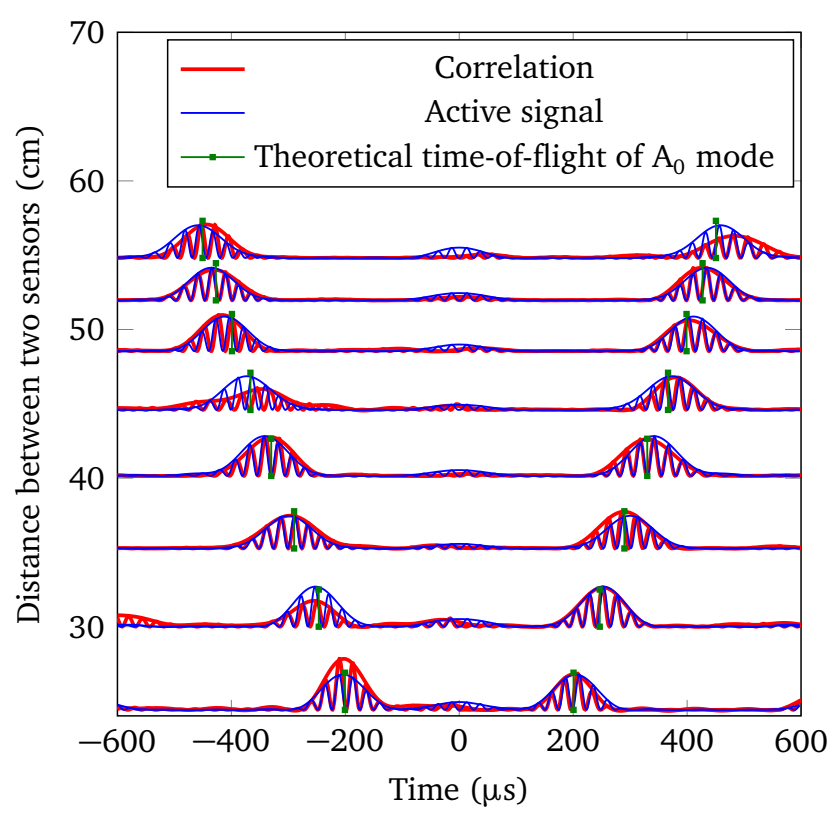

(a)

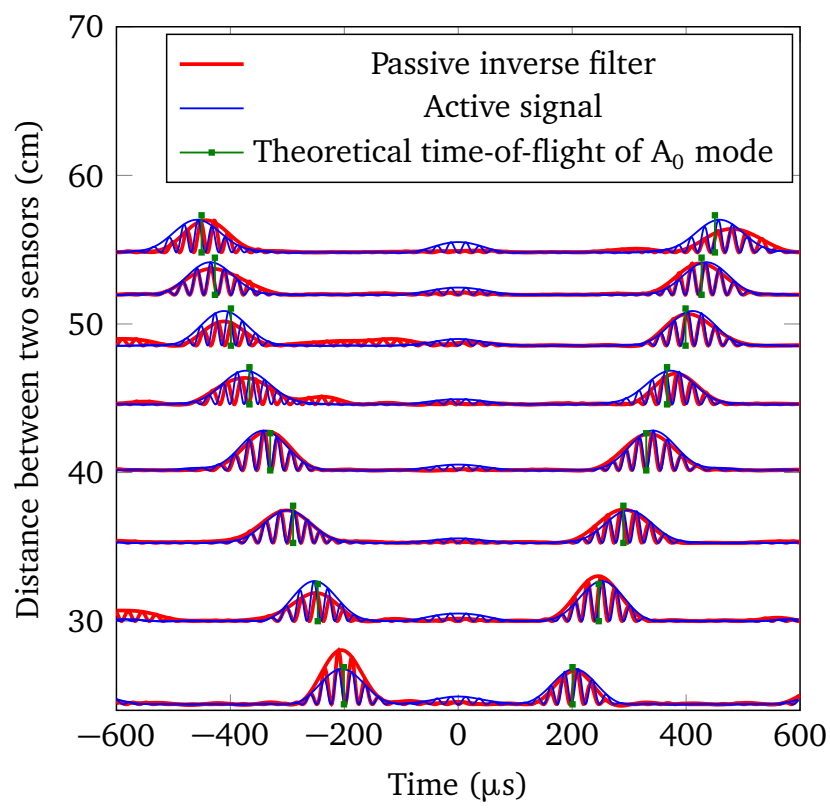

(b)

FIG. 5. (Color online) Comparison between active signals and (a) cross-correlation (b) passive inverse filter.

wave tomography. This is the subject of the following section.

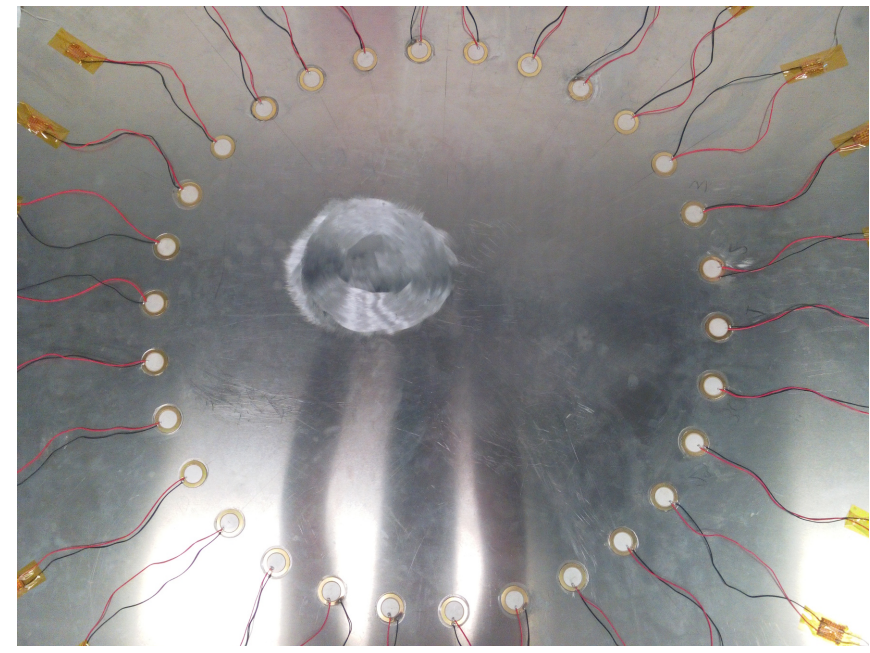

FIG. 6. (Color online) Defect position.

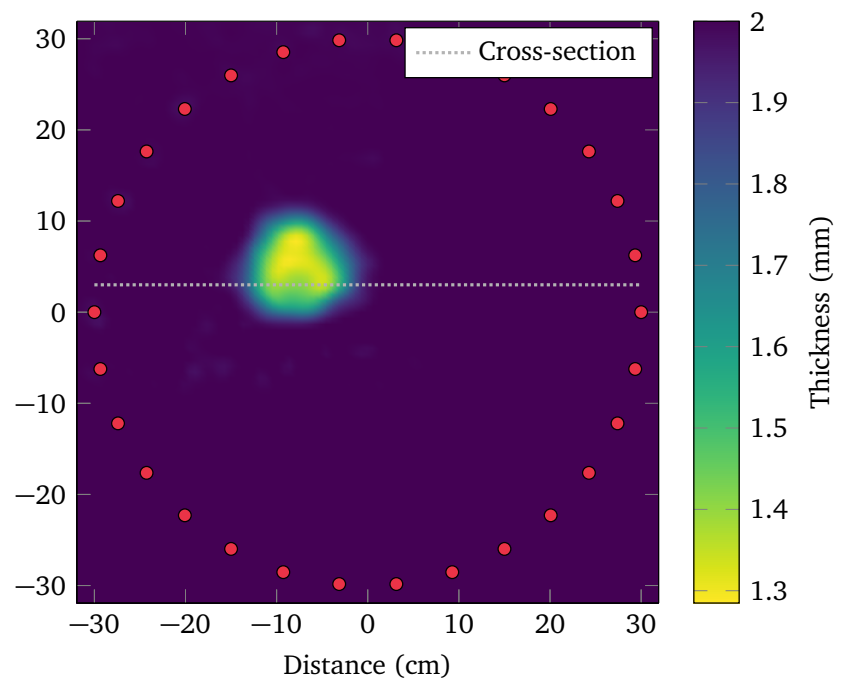

FIG. 7. (Color online) Reference measured thanks to an immersion ultrasonic scan.

\section{Passive images with guided wave tomography}

In order to test passive tomography a defect was created into the zone of interest (see the figure 6) by machining locally the aluminum plate. A reference of the defect has been obtained thanks to an immersion ultrasonic scan, such a scan being significantly more precise than a global method such as guided wave tomography. The reference is plotted on figure 7 .

During the acquisition of the ambient noise, an effort has been made to respect, as a first step, the equipartition hypothesis as much as possible. This means that the compressed air jet was moved on the whole surface in order to spend an equivalent time on each surface areas. Passive tomography is first computed without the use of the "variable relaxation". The result is visible fig- 


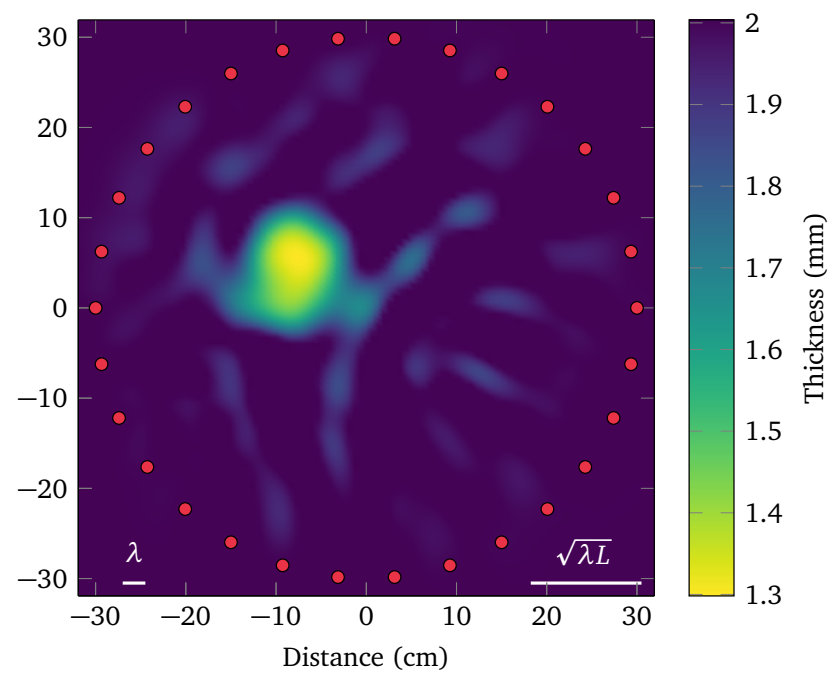

(a)

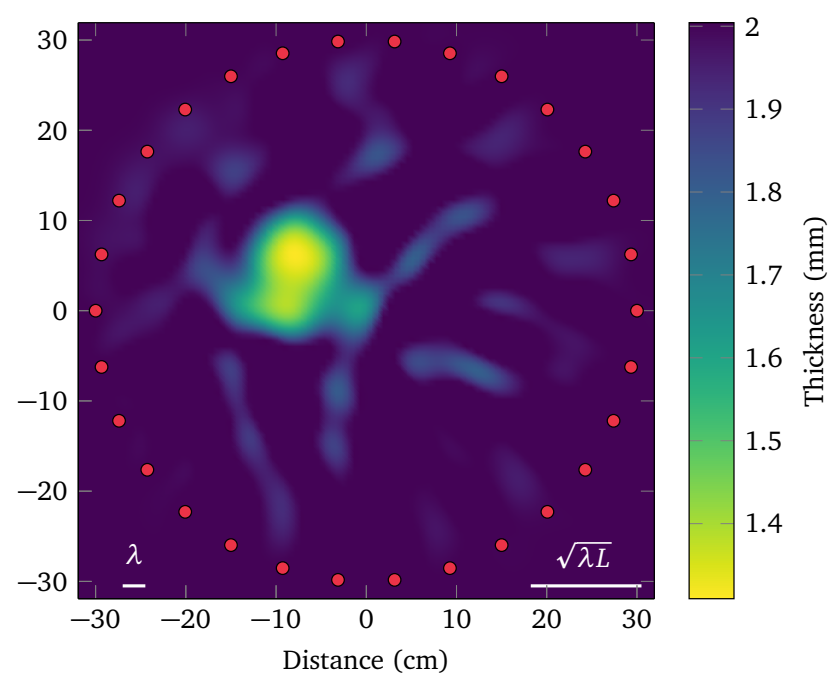

(b)

FIG. 8. (Color online) Passive guided wave tomography without regularization for (a) the cross-correlation and (b) the passive inverse filter.

ure 8(a) for the cross-correlation and figure $8(\mathrm{~b})$ for the passive inverse filter. We can see on both images typical aliasing due to the fact that we use undersampled data (see (Druet et al., 2019) for more details). Moreover, both passive tomography images seem to be of the same quality.

In order to compensate the aliasing effect, the "variable relaxation" is added in the guided wave tomography process, as explained section IIB. The results are presented figure 9, image (a) for the cross-correlation and image (b) for the passive inverse filter. Once again, both images are of same quality but this time the aliasing effect

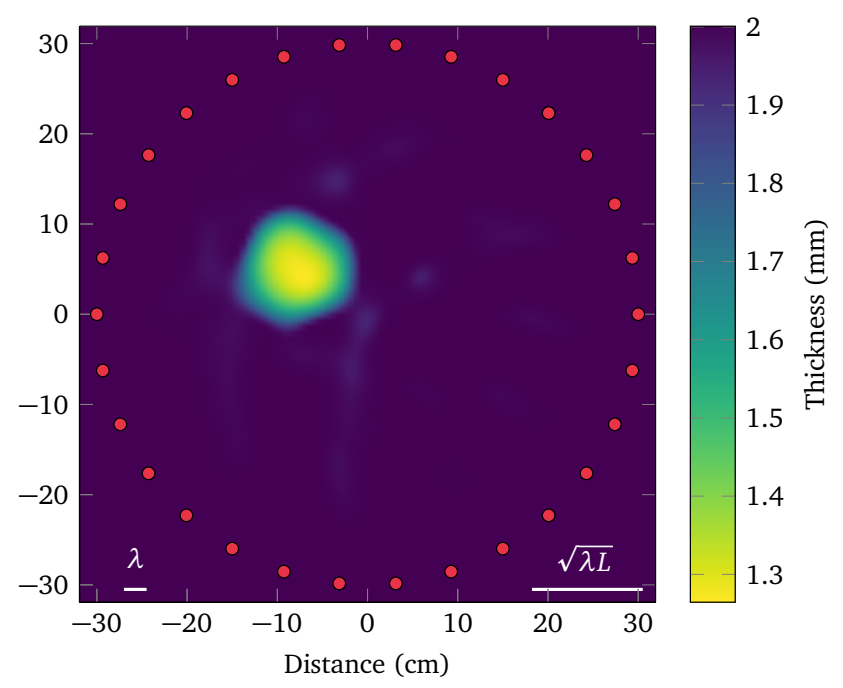

(a)

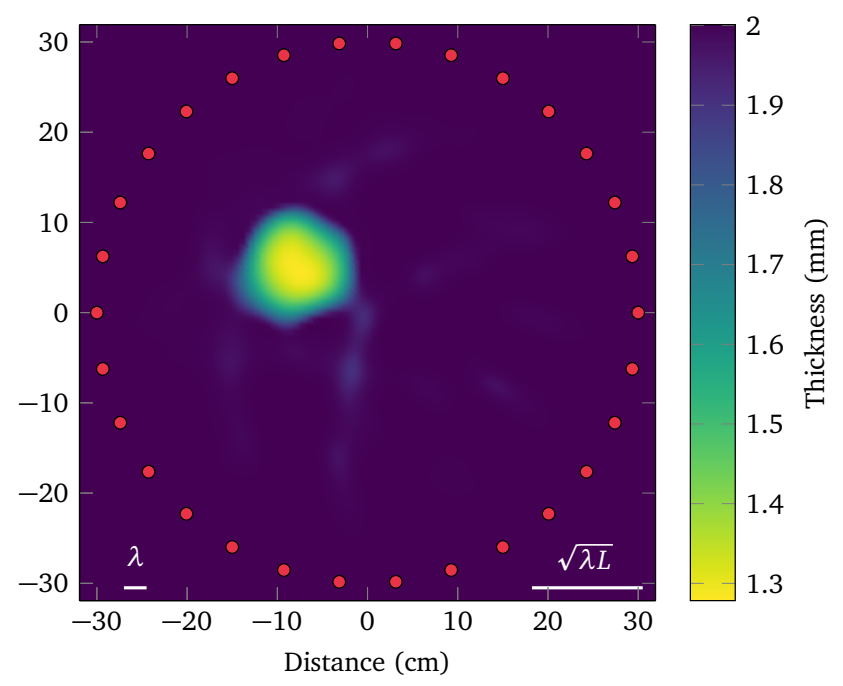

(b)

FIG. 9. (Color online) Passive guided wave tomography with regularization for (a) the cross-correlation and (b) the passive inverse filter.

is significantly reduced. The background of the image is very clean so that the defect clearly appears in the inspected area. Moreover, compared to the reference, the localization of the defect is well identified and the geometry is almost fully reconstructed. In order to compare more quantitatively the results, we propose cross-sections of the images on figure 10 . The position of the crosssection is visible on the reference (figure 7 ). The small variations within the defect visible in the reference of the figure 7 are the only thing that is not reconstructed by the passive tomography algorithm. This is because the 


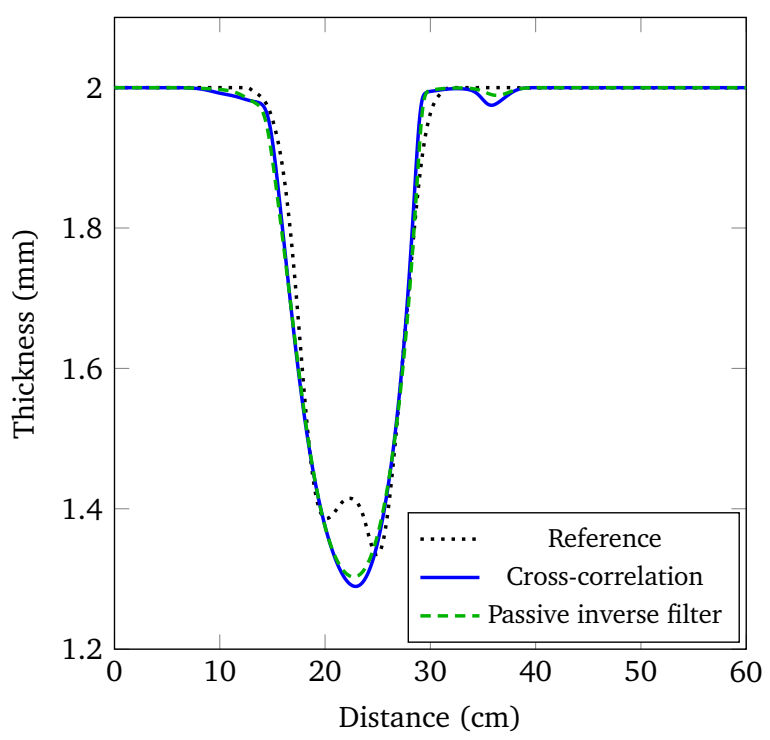

FIG. 10. (Color online) Cross-sections of the passive guided wave tomographies with regularization.

working wavelength is too large to be able to reconstruct such a variation.

Passive tomography gives really good results with an ambient noise which respects as much as possible the equipartition condition but this condition is not always respected in real industrial applications. That is why we now study a case which is closer to reality. Indeed, this time the compressed air jet is only moved onto left-half of the plate area. This is supposed to throw the energy repartition off balance. The passive guided wave tomographies corresponding to that configuration are presented figure 11(a) for the cross-correlation and figure 11(b) for the passive inverse filter. The results are of the same quality as the ones for the equipartition case (figure 9 ). This can be explained by the fact that the aluminum plate is a very reverberant structure compensating for the bad equipartition of the ambient noise.

To conclude, passive guided wave tomography gives a really good image of corrosion flaws whether it be with the cross-correlation or with the passive inverse filter. However, as explained in section II A, the passive inverse filter should give better results than the cross-correlation in cases where the noise does not fill the equipartition hypothesis. The structure studied in this paper did not permit to break this hypothesis and to illustrate this fact. Future studies in this direction seem interesting.

\section{CONCLUSIONS}

In this paper were presented imaging results of corrosion-like flaws using a passive guided wave tomography algorithm. To do so, two passive data reconstruction were compared: the usual ambient noise cross-correlation and the passive inverse filter. Even though the results are very similar for both methods, the passive inverse filter

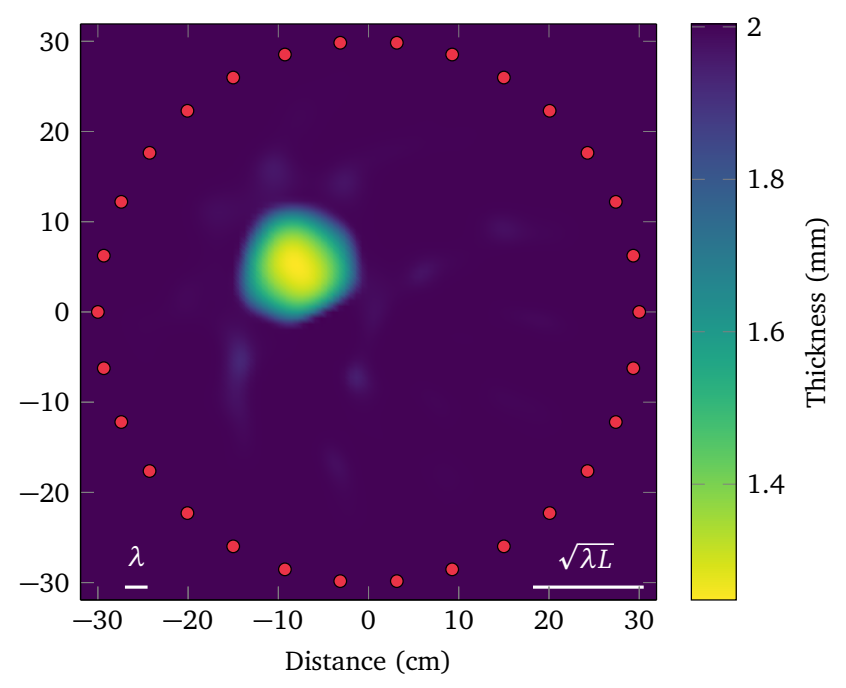

(a)

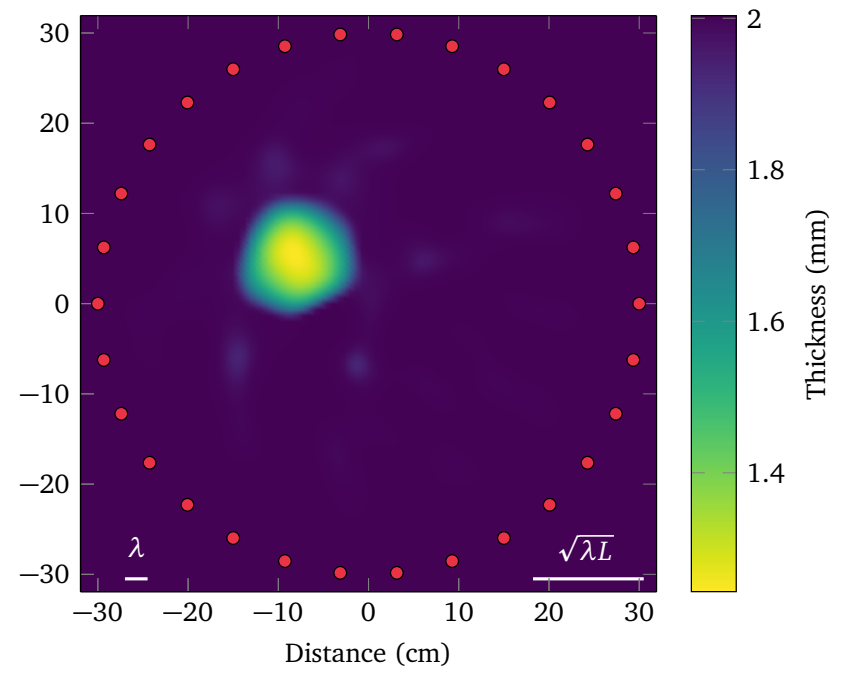

(b)

FIG. 11. (Color online) Passive guided wave tomography with regularization for (a) Correlation (b) Passive inverse filter. Non equipartition case.

seem to be a promising method as its working hypotheses seem to be weaker. Indeed, the passive inverse filter can be seen as a regularized version of the cross-correlation and thus, it should be more robust. A thorough study is yet to be done. Furthermore, the passive inverse filter is naturally well-suited for configurations using many sensors, which is the case of tomography algorithms.

This study was done using classical piezoelectric transducers, but the use of passive algorithms allow the use of sensors not able to emit waves, for example the fiber BRAGG gratings. These sensors are really interesting because of their low intrusiveness in the context 
of SHM and our current work focus on the use of fiber BRAGG gratings.

The application presented in this paper is a rather well-controlled laboratoy configuration. We plan on applying the method to real structures, using the ambient noise coming from their environment to image it.

Auger, F., and Flandrin, P. (1995). "Improving the readability of time-frequency and time-scale representations by the reassignment method," IEEE Transactions on Signal Processing 43(5), 1068-1089, doi: 10.1109/78.382394.

Campillo, M., and Paul, A. (2003). "Long-range correlations in the diffuse seismic coda," Science 299, 547-549.

Chehami, L., Moulin, E., de Rosny, J., Prada, C., Bou Matar, O., Benmeddour, F., and Assaad, J. (2014). "Detection and localization of a defect in a reverberant plate using acoustic field correlation," Journal of Applied Physics 115, 104901.

Croxford, A., Moll, J., Wilcox, P., and Michaels, J. (2010). "Efficient temperature compensation strategies for guided wave structural health monitoring," Ultrasonics 50, 517-528.

Croxford, A., Wilcox, P., Drinkwater, B., and Konstantinidis, G. (2007). "Strategies for guided-wave structural health monitoring," Proc. R. Soc. A 463, 2961-2981.

Davy, M., de Rosny, J., and Besnier, P. (2016). "Green's function retrieval with absorbing probes in reverberating cavities," Phys. Rev. Lett. 116, 213902, http://link.aps.org/doi/ 10.1103/PhysRevLett.116.213902, doi: 10.1103/PhysRevLett. 116. 213902.

Druet, T., Chapuis, B., Jules, M., Laffont, G., and Moulin, E. (2018). "Passive guided waves measurements using fiber Bragg gratings sensors," The Journal of the Acoustical Society of America 144(3), 1198-1202, https://doi.org/10.1121/1.5054015, doi: $10.1121 / 1.5054015$.

Druet, T., Tastet, J.-L., Chapuis, B., and Moulin, E. (2019). "Autocalibration method for guided wave tomography with undersampled data," Wave Motion 89, 265-283, https://doi.org/10. 1016/j. wavemoti.2019.04.002.

Duvall Jr, T. L., Jeffferies, S., Harvey, J., and Pomerantz, M. (1993). "Time-distance helioseismology," Nature 362(6419), 430.

Gallot, T. (2010). "Acoustical imaging in reverberating medias [in French]," Theses, Université de Grenoble, https://tel. archives-ouvertes.fr/tel-00606012.

Gallot, T., Catheline, S., Roux, P., and Campillo, M. (2012). "A passive inverse filter for green's function retrieval," The Journal of the Acoustical Society of America 131(1), EL21-EL27.

Garnier, J., and Papanicolaou, G. (2016). Passive imaging with ambient noise (Cambridge University Press).

Huthwaite, P. (2014). "Evaluation of inversion approaches for guided wave thickness mapping," Proceedings of the Royal Society A 470, 20140063.

Huthwaite, P. (2016a). "Eliminating incident subtraction in diffraction tomography," Proceedings of the Royal Society of London A: Mathematical, Physical and Engineering Sciences 472(2195), http://rspa.royalsocietypublishing.org/ content/472/2195/20160707, doi: 10.1098/rspa.2016.0707.

Huthwaite, P. (2016b). "Guided wave tomography with an improved scattering model," Proceedings of the Royal Society of London A: Mathematical, Physical and Engineering Sciences 472(2195), http://rspa.royalsocietypublishing.org/ content/472/2195/20160643, doi: 10.1098/rspa.2016.0643.

Huthwaite, P. (2016c). "Improving accuracy through density correction in guided wave tomography," Proceedings of the Royal Society of London A: Mathematical, Physical and Engineering Sciences 472(2186), http://rspa.royalsocietypublishing. org/content/472/2186/20150832, doi: 10.1098/rspa.2015. 0832.

Huthwaite, P., and Simonetti, F. (2013). "High-resolution guided wave tomography," Wave Motion 50, 979-993.

Huthwaite, P., Zwiebel, A. A., and Simonetti, F. (2013). "A new regularization technique for limited-view sound-speed imaging,"
IEEE Transactions on Ultrasonics Ferroelectrics and Frequency Control 60(3), 603-613.

Larose, E., Roux, P., and Campillo, M. (2007). "Reconstruction of Rayleigh-Lamb dispersion spectrum based on noise obtained from an air-jet forcing," Journal of the Acoustical Society of America 122, 3437-3444.

Liu, C., Harley, J. B., Bergés, M., Greve, D. W., and Oppenheim, I. J. (2015). "Robust ultrasonic damage detection under complex environmental conditions using singular value decomposition," Ultrasonics 58, 75-86, http://www.sciencedirect. com/science/article/pii/S0041624X14003515, doi: http:// $\mathrm{dx}$.doi.org/10.1016/j.ultras.2014.12.005.

Lobkis, O., and Weaver, R. (2001). "On the emergence of the Green's function in the correlations of a diffuse field," Journal of the Acoustical Society of America 6, 3011-3017.

Michaels, J. E. (2008). "Detection, localization and characterization of damage in plates with an in situ array of spatially distributed ultrasonic sensors," Smart Materials and Structures $\mathbf{1 7}(3), 035035$.

Putkis, O., and Croxford, A. J. (2013). "Continuous baseline growth and monitoring for guided wave shm," Smart Materials and Structures 22(5), 055029.

Quaegebeur, N., Ostiguy, P., and Masson, P. (2014). "Correlationbased imaging technique for fatigue monitoring of riveted lapjoint structure," Smart Materials and Structures 23, 055007 (10pp).

Roux, P., Kuperman, W., Colosi, J., Cornuelle, B., Dushaw, B., Dzieciuch, M., Howe, B., Mercer, J., Munk, W., Spindel, R., and Worcester, P. (2004). "Extracting coherent wave fronts from acoustic ambient noise in the ocean," The Journal of the Acoustical Society of America 116(4), 1995-2003.

Sabra, K., Srivastava, A., Lanza di Scalea, F., Bartoli, I., Rizzo, P., and Conti, S. (2008). "Structural health monitoring by extraction of coherent guided waves from diffuse fields," Journal of the Acoustical Society of America 132, 8-13.

Sabra, K., Winkel, E., Bourgoyne, D., Elbing, B., Ceccio, S., Perlin, M., and Dowling, D. (2007a). "Using cross correlations of turbulent flow-induced ambient vibrations to estimate the structural impulse response. application to structural health monitoring," Journal of the Acoustical Society of America 121, 1987-1995.

Sabra, K. G., Conti, S., Roux, P., and Kuperman, W. (2007b). "Passive in vivo elastography from skeletal muscle noise," Applied physics letters 90(19), 194101.

SAE International (2013). "Guidelines for implementation of structural health monitoring on fixed wing aircraft," ARP6461.

Seydoux, L., de Rosny, J., and Shapiro, N. M. (2017). "Preprocessing ambient noise cross-correlations with equalizing the covariance matrix eigenspectrum," Geophysical Journal International 210(3), 1432-1449.

Shi, F., and Huthwaite, P. (2018). "Ultrasonic wave-speed diffraction tomography with undersampled data using virtual transducers," IEEE Transactions on Ultrasonics, Ferroelectrics, and Frequency Control .

Stehly, L., Campillo, M., Froment, B., and Weaver, R. (2008). "Reconstructing green's function by correlation of the coda of the correlation (C3) of ambient seismic noise," Journal of the Geophysical Research .

Yu, L., and Giurgiutiu, V. (2008). "In situ 2-D piezoelectric wafer active sensors arrays for guided wave damage detection," Ultrasonics 48, 117-134.

Zhao, X., Gao, H., Zhang, G., Ayhan, B., Yan, F., Kwan, C., and Rose, J. (2007). "Active health monitoring of an aircraft wing with embedded piezoelectric sensor/actuator network: I. defect detection, localization, and growth monitoring," Smart Materials and Structures 16, 1208-1217. 
